\title{
First Total Synthesis of Prorepensin with a Bis-Geranylated Chalcone
}

\author{
Eun Mi Jung and Yong Rok Lee* \\ School of Chemical Engineering and Technology, leungnam Universitv, Gleongsan 712-749, Korea \\ "E-mail: vrleéduvu ac.hr \\ Received September 2, 2009, Accepted September 8, 2009
}

\begin{abstract}
The first total synthesis of naturally occurring prorepensin with a bis-geranylated chalcone has been achieved by a convergent sequence. The key strategy involved in the synthesis of prorepensin was chalcone formation by aldol condensation of the corresponding geranylated acetophenone with geranylated benzaldehyde.
\end{abstract}

Key Words: Prorepensin. Geranylated chalcone, Aldol reaction

\section{Introduction}

Geranylated chalcones are an abundant subclass of flavo-

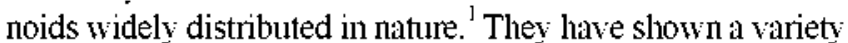
of biological properties including antibacterial. ${ }^{2}$ antifungal. ${ }^{3}$ antitumor, ${ }^{4}$ antimetastatic. ${ }^{5}$ cancer chemopreventive, ${ }^{6}$ antiHIV. anti-yasoconstriction. ${ }^{8}$ and antidiabetic activities ${ }^{8}$ and have been used in traditional medicines. ${ }^{\text {(C) }}$ Interestingly, it was reported that the presence of the geranyl group in chalcone<smiles>CC(C)=CCC/C(C)=C/Cc1cc(C(=O)/C=C/c2cc(O)c(O)c(C/C=C(\C)CCC=C(C)C)c2)ccc1O</smiles>

Figure 1

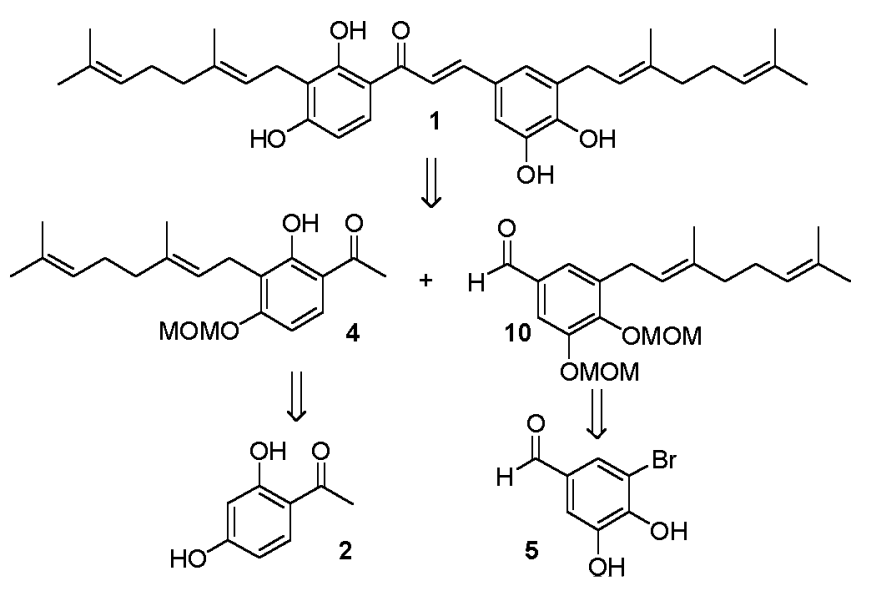

Scheme 1 skeleton leads to a remarkable increase in corresponding bioactivities. ${ }^{11}$ These important biological activities and properties have aroused interest in the synthesis of naturally occurring geranylated chalcones. As part of a systematic search for new bioactive lead compounds from African medicinal plants. prorepensin (1) was separately isolated from Dorstenia prorepens and D. picta (Fig. 1).

This range of important biological activities and properties has stimulated research into the total synthesis of prorepensin (1). In particular, no total sy'nthesis of naturally occurring prorepensin (1) with a bis-geranylated chalcone has been reported thus far

Recently, we have developed efficient and useful synthetic routes for preparing pyranochalcones. ${ }^{13}$ As part of an ongoing study into the efficacy of such synthetic approaches. the synthesis of prorepensin (1) with the bis-geranylated chalcone skeleton has been attempted. We report herein the first total synthesis of the naturally occurring prorepensin (1) with a bis-geranylated chalcone.

\section{Results and Discussion}

Scheme 1 shows the retrosynthetic strategy for the synthesis of prorepensin (1). The synthesis of the chalcone structure is readily accomplished through condensation two suitable partners. 3-gernaylated acetophenone 4 and 3-geranylated benzaldehyde 10 . Compound $t$. as the left half. could be generated starting from 2,4-dilydrosyacetophenone (2) by a geranylation reaction and selective mono protection of the two phenol groups as a MOM ether. Compound 10. as the right lalf, could be prepared from 3-bromo-4.5-dihy droxybenzaldehyde (5).

The synthesis of intermediate 4 was first attempted starting from commercially available 2.4-dihydroxyacetophenone (2). as shown in Scheme 2. Treatment of 2.4-dihydroxyacetophenone (2) and geranyl bronide with $\mathrm{KOH}$ in methanol at room

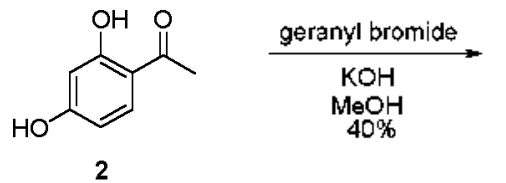

2<smiles>CC(=O)c1ccc(O)c(C/C=C(\C)CCC=C(C)C)c1O</smiles>

3

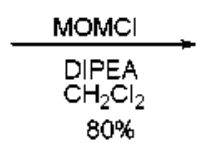

$80 \%$<smiles>COc1ccc(C(C)=O)c(O)c1C/C=C(\C)CCC=C(C)C</smiles>

Scheme 2 
<smiles>O=Cc1cc(O)c(O)c(NO[Sn](=O)[O-])c1</smiles>

5<smiles>COc1cc(C=O)cc(Br)c1OC</smiles><smiles>COc1cc(CO)cc(C/C=C(\C)CCC=C(C)C)c1OC</smiles><smiles>COc1cc(CO)cc(Br)c1OC</smiles>

7

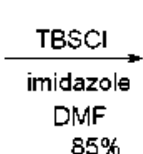

$85 \%$<smiles>COc1cc(COC(C)C)cc(Br)c1OC</smiles>

8
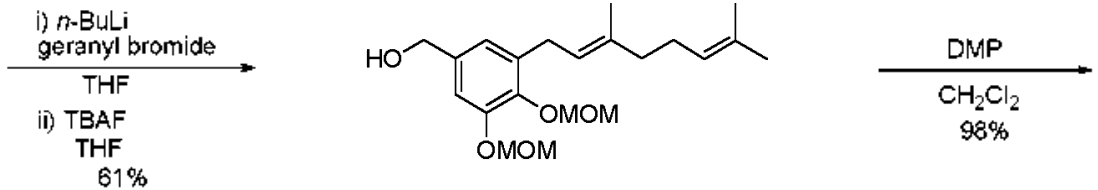<smiles>COc1cc(C=O)cc(C/C=C(\C)CCC=C(C)C)c1OC</smiles>

10

Scheme 3

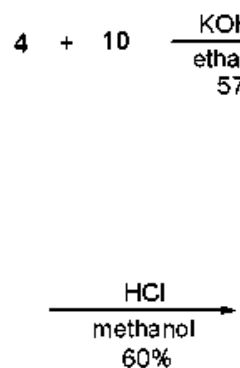

$60 \%$<smiles>CC(C)=CCC/C(C)=C/Cc1cc(/C=C/C(=O)c2ccc(O)c(C/C=C(\C)CCC=C(C)C)c2O)cc(O)c1O</smiles>

Scheme 4 temperature for $2+\mathrm{h}$ afforded gerany lated product 3 in $40 \%$ yield. ${ }^{l 4}$ Protection of 3 with 1.0 equiv of methoxymethyl chloride in the presence of $\mathrm{N} . \mathrm{N}$-diisopropy lethylamine gave 4 in $80 \%$ yield. The selective mono methoxymethylation of compound 4 was confirmed by analysis of the ${ }^{1} \mathrm{H}$ NMR spectnum. The signal for the hydroxyl proton in + was observed as a singlet associated with a hy drogen bond to a carbonyl group at $\hat{\delta} 12.75$ ppm. A methoxy signal of the MOM ether was observed as a singlet at ò $3.45 \mathrm{ppm}$.

As shown in Scheme 3, the synthesis of intermediate $\mathbf{1 0}$ as the right half was begun with the readily available 3-bromo-4,5dihydroxỵbenzaldehyde (5). Reaction of 5 with 2.2 eq of methoxymethyl chloride in the presence of sodium hydride afforded compound 6 in $85 \%$ yield. Reduction of 6 with sodium borohydride in methanol gave benzylic alcohol 7 in $90 \%$ yield. Treatment of 7 with TBSCl in the presence of imidazole gave silyl ether 8 in $85 \%$ yield. Compound 9 was produced in $61 \%$ ( 2 steps) yield by halogen-metal exchange and alkylation reaction using $n$-BuLi with gerany l bromide in $\mathrm{THF}^{15}$ followed by cleavage of the silyl ether with TBAF in THF. Oxidation of 9 with Dess-Martin periodinane (DMP) in metlyy lene clloride gave the left half of intermediate 10 in $98 \%$ yield. ${ }^{16}$

To complete the total synthesis of natural product prorepensin (1). an aldol reaction was next attempted (Scheme 4). Treatment of 4 with aryl benzaldelyde 10 in ethanolic $\mathrm{KOH}$ at room temperature for $48 \mathrm{~h}$ provided the desired chalcone 11 in $57 \%$ yield. Deprotection of 11 with concentrated $\mathrm{HCl}$ in methanol at room temperature for 12 h gave prorepensin (1) in
$60 \%$ yield. The spectral data of synthetic material 1 were in agreement with those previously reported. ${ }^{\text {?a }}$

In conclusion, the first total syinthesis of prorepensin (1) has been accomplished by a convergent sequence. The key strategy for the synthesis of prorepensin (1) was chalcone formation by an aldol reaction of geranylated acetophenone 4 and gerany lated benzaldehyde 10. prepared separately from 2.4-dihy'droxyacetophenone (2) and 3-bromo-4.5-dihydroxybenzyl alcohol (5).

\section{Experimental Section}

All experiments were carried out in a nitrogen atmosphere. Merck pre-coated silica gel plates (Art. 5554) with a fluorescent indicator were used for analytical TLC. Flash column chromatography was performed using silica gel $9385 .{ }^{1} \mathrm{H}$ and ${ }^{13} \mathrm{C}$ NMR spectra were recorded on a Bruker Model ARX ( 300 and $75 \mathrm{MHz}$, respectively) spectrometer in $\mathrm{CDCl}_{3}$ as the solvent chemical shift. The IR spectra were recorded on a Jasco FTIR 5300 spectrophotometer. The HRMS spectra were carried out at the Korea Basic Science Institute.

3-Geranyl-2,4-dihydioxyacetophenone (3). To a solution of 2.4-dihydrosyacetophenone (2) $(2.450 \mathrm{~g} .16 .1 \mathrm{mmol})$ in methanol $(50 \mathrm{~mL})$ was added geranyl bromide $(3.846 \mathrm{~g} .17 .7$ nunol) and anhydrous potassiun hydroxide $(9.034 \mathrm{~g}, 161.0$ nmol). The reaction mixture was stirred at room temperature for $24 \mathrm{~h}$. Evaporation of methanol. addition of $2 \mathrm{~N} \mathrm{HCl}$ solution $(50 \mathrm{~mL})$, and extraction with ethyl acetate $(3 \times 100 \mathrm{~nL})$, 
washing with brine $(100 \mathrm{~mL})$, drying over anhydrous $\mathrm{MgSO}_{4}$. and removal of the solvent followed by flash column chromatography on silica gel using hexane/ethylacetate (7:1) gave product $3(1.875 \mathrm{~g} .40 \%)$ as a solid: $\mathrm{mp} 101-102^{\circ} \mathrm{C}$; ${ }^{\mathrm{l}} \mathrm{H}$ NMR $\left(300 \mathrm{MHz}, \mathrm{CDCl}_{3}\right) \delta 13.09$ (1H. s). 7.52 (1H. d. $J=9.0 \mathrm{~Hz}$ ). $6.36(1 \mathrm{H}, \mathrm{d} . J=9.0 \mathrm{~Hz}) .5 .25(1 \mathrm{H}, \mathrm{t}, J=6.6 \mathrm{~Hz}), 5.03(1 \mathrm{H}, \mathrm{t}$, $J=6.3 \mathrm{~Hz}$ ). $3.43(2 \mathrm{H}, \mathrm{d}, J=6.3 \mathrm{~Hz}) .2 .5+(3 \mathrm{H}, \mathrm{s}) .2 .15-2.01$ (4H. m) $1.80(3 \mathrm{H}, \mathrm{s}) .1 .65(3 \mathrm{H}, \mathrm{s}) .1 .57(3 \mathrm{H} . \mathrm{s}):{ }^{13} \mathrm{C} \mathrm{NMR}(75$ $\left.\mathrm{MHz}, \mathrm{CDCl}_{3}\right) \delta 202.8,162.7,161.8,137.3 .131 .4 .130 .0$. $124.0,121.3 .114 .7,113.4 .107 .6,39.6,26.4,25.9 .25 .5 .21 .4$. 17.5. 16.0: $\mathrm{R}(\mathrm{KBr}) 3167,2969.1624,1449.1374,1274$. 1163, $1055,915,792,712,609 \mathrm{~cm}^{-1}:$ HRMS $m / z\left(\mathrm{M}^{+}\right)$calcd for $\mathrm{C}_{18} \mathrm{H}_{24} \mathrm{O}_{3}: 288.1725$. Found: 288.1722 .

1-[3-(3,7-Dimethy]-octa-2,6-dieny])-2-hydroxy-4-methoxymethoxyphenyl] ethanone (4). Methoxymethyl chloride $(0.335 \mathrm{~g}$. $+.2 \mathrm{mmol}$ ) was added to a solution of $3(1.20 \mathrm{~g} .4 .2 \mathrm{mmol})$ and $\mathrm{N}, \mathrm{N}$-diisopropylethylamine $(3.361 \mathrm{~g} .26 .0 \mathrm{mmol})$ in dry $\mathrm{CH}_{2} \mathrm{Cl}_{2}(20 \mathrm{~mL})$. The reaction mixture was stirred at room temperature for $4 \mathrm{~h}$ and then water $(30 \mathrm{~mL})$ was added. The reaction mixture was extracted with $\mathrm{CH}_{2} \mathrm{Cl}_{2}(3 \times 30 \mathrm{~mL})$. The combined organic extracts were washed with saturated $\mathrm{NH}_{4} \mathrm{Cl}$ solution $(30 \mathrm{~mL})$ and evaporated in vacuo. Flash chromatography on silica gel with hexane/EtOAc (6:1) afforded 4 ( $1.106 \mathrm{~g} .80 \%$ ) as an oil: ${ }^{1} \mathrm{H} \mathrm{NMR}\left(300 \mathrm{MHz}, \mathrm{CDCl}_{3}\right) \hat{o} \mathrm{I} 2.75$ $(1 \mathrm{H}, \mathrm{s}) .7 .55(1 \mathrm{H} . \mathrm{d} . J=9.0 \mathrm{~Hz}), 6.62(1 \mathrm{H}, \mathrm{d}, J=9.0 \mathrm{~Hz}) .5 .24$ $(2 \mathrm{H} . \mathrm{s}) .5 .20(\mathrm{lH}, \mathrm{t} J=6.3 \mathrm{~Hz}) .5 .04(1 \mathrm{H}, \mathrm{t} . J=6.3 \mathrm{~Hz}) .3 .45$ $(3 \mathrm{H}, \mathrm{s}) .3 .37(2 \mathrm{H}, \mathrm{d} . J=6.3 \mathrm{~Hz}) .2 .54(3 \mathrm{H}, \mathrm{s}) .2 .06-1.91(4 \mathrm{H}$. iin), 1.77 (3H. s), 1.61 (3H. s), 1.54 (3H. s): ${ }^{13} \mathrm{C} \operatorname{NMR}(75$ $\left.\mathrm{MHz}, \mathrm{CDCl}_{3}\right) \delta 202.7,161.7,160.5,134.8,130.8,129.6$. $124.2,121.7 .117 .9,114.5 .104 .6,93.5,55.8 .39 .6 .26 .5 .25 .9$. 25.4. $21.5,17.3,15.8$ : IR (neat) $2919,1629,1496,1419$. 1370. $1260,1157.1054,988.797 .688 \mathrm{~cm}^{-1}$; HRMS $m / z$ (M) calcd for $\mathrm{C}_{21} \mathrm{H}_{2} \mathrm{O}_{4}: 332.1988$. Found: 332.1986 .

3-Bromo-4,5-dimethoxymethoxybenzaldehyde (6). To a stirred solution of 5-bromo-3,4-dihydroxybenzaldehyde (5) $(2.0 \mathrm{~g} .9 .2 \mathrm{mmol})$ in dry DMF $(40 \mathrm{~mL})$ at $0{ }^{\circ} \mathrm{C}$ was added sodium hydride $(2.20 \mathrm{~g} .92 .0 \mathrm{mmol})$ and stirred for $10 \mathrm{~min}$. To the reaction mixture was added methoxymethyl chloride (MOMCl) (1.840 g. $23.0 \mathrm{mmol}$ ) slowly and continued the reaction for $1 \mathrm{~h}$. The reaction was quenched by the addition of water $(30 \mathrm{~mL})$ and extracted with EtOAc $(3 \times 50 \mathrm{~mL})$. The organic layer was washed with saturated $\mathrm{NH}_{4} \mathrm{Cl}$ solution ( 30 $\mathrm{mL}$ ) and then dried over anhydrous $\mathrm{Na}_{2} \mathrm{SO}_{4}$ and concentrated under reduced pressure to afford crude residue. Flash column chromatography on silica gel with hexane/EtOAc (5:1) afforded $6(2.380 \mathrm{~g} .85 \%)$ as an oil: ${ }^{l} \mathrm{H}$ NMR $\left(300 \mathrm{MHz} . \mathrm{CDCl}_{3}\right)$ oे 9.80 (1H. s). 7.70 (1H, s). 7.57 (1H. s), 5.26 (2H. s). 5.22 (2H, s). $3.6 \mathrm{I}(3 \mathrm{H}, \mathrm{s}) .3 .46(3 \mathrm{H}, \mathrm{s}):{ }^{13} \mathrm{C} \mathrm{NMR}\left(75 \mathrm{MHz} \mathrm{CDCl}_{3}\right)$ ò 189.5 . 151.1, 149.2, 133.1. 128.7. 118.3, 115.2, 98.8, 95.1, 58.0, 56.5: IR (neat) $2956,2833,1696,1568,1478,1380,1276,1158$. 1082. $1011,936,745.669 \mathrm{~cm}^{-1}$ : HRMS $m / z\left(\mathrm{M}^{-}\right)$calcd for $\mathrm{C}_{11} \mathrm{H}_{13} \mathrm{BrO}_{3}: 303.9946$. Found: 303.9948 .

3-Bromo-4,5-dimethoxymethoxybenzyl alcohol (7). To a stirred solution of $6(1.0 \mathrm{~g}, 3.3 \mathrm{mmol})$ in methanol $(30 \mathrm{~mL})$ at $0{ }^{\circ} \mathrm{C}$ was added sodium borohydride $(0.186 \mathrm{~g} .4 .9 \mathrm{mmol})$ in small portions and continued the reaction for $1 \mathrm{~h}$. The reaction mixture was quenched by the addition of water $(30 \mathrm{~mL})$ and extracted with EtOAc $(3 \times 40 \mathrm{~mL})$. The organic layer was washed with saturated $\mathrm{NH}_{4} \mathrm{Cl}$ solution $(40 \mathrm{~mL})$ and then dried over anhydrous $\mathrm{Na}_{2} \mathrm{SO}_{4}$. and concentrated under reduced pressure to afford cnude residue. Flash column chromatography on silica gel with hexane/EtOAc $(3: 1)$ afforded $7(0.90 \mathrm{~g} .90$ $\%$ ) as an oil: ${ }^{1} \mathrm{H} \mathrm{NMR}\left(300 \mathrm{MHz}, \mathrm{CDCl}_{3}\right)$ ò 7.17 (lH. s). 7.05 (1H. s). 5.15 (2H. s). $5.13(2 \mathrm{H}, \mathrm{s}) .4 .54(2 \mathrm{H}, \mathrm{s}), 3.62(3 \mathrm{H}, \mathrm{s})$, $3.45(3 \mathrm{H}, \mathrm{s}):{ }^{13} \mathrm{CNMR}\left(75 \mathrm{MHz}, \mathrm{CDCl}_{3}\right) \delta 150.9,143.2,138.4$. $124.5,117.8 .114 .2,98.8 .95 .2,64.1,57.9 .56 .4$ : IR (neat) 3430,2935 . 1569. 1481. 1275. 1157, 1006, 949,852.676 $\mathrm{cm}^{-1}$; HRMS $m / z\left(\mathrm{M}^{-}\right)$calcd for $\mathrm{C}_{11} \mathrm{H}_{15} \mathrm{BrO}_{5}: 306.0103$. Found: 306.0103 .

(3-Bromodimethoxymethoxybenzyloxy)-t-butyldimethylsilane (8). To a stirred solution of $7(0.80 \mathrm{~g} .2 .6 \mathrm{numol})$, in dry $\mathrm{DMF}(10 \mathrm{~mL})$ at $0{ }^{\circ} \mathrm{C}$ was added imidazole $(0.443 \mathrm{~g} .6 .5$ mmol) and stirred for $10-15 \mathrm{~min}$. TBSCl $(0.471 \mathrm{~g} .3 .1 \mathrm{mmol})$ was added carefully and the reaction nixture was allowed to stir at room temperature for $10 \mathrm{~h}$. The reaction misture was quenched by the addition of water ( $30 \mathrm{~nL}$ ) and extracted with EtOAc $(3 \times 30 \mathrm{~mL})$. The organic layer was washed with saturated $\mathrm{NH}_{4} \mathrm{Cl}$ solution ( $30 \mathrm{~mL}$ ) and then dried over anhydrous $\mathrm{Na}_{2} \mathrm{SO}_{4}$ and concentrated under reduced pressure to give crude residue. Flash column chromatography on silica gel with hexane/EtOAc (4:1) afforded $8(0.932 \mathrm{~g} .85 \%)$ as an oil: ${ }^{1} \mathrm{H}$ NMR $\left(300 \mathrm{MHz}, \mathrm{CDCl}_{3}\right) \delta 8.1+(\mathrm{lH}, \mathrm{s}) .7 .06(\mathrm{lH}, \mathrm{s})$. $5.15(2 \mathrm{H} . \mathrm{s}) .5 .1+(2 \mathrm{H}, \mathrm{s}) .+61(2 \mathrm{H}, \mathrm{s}), 3.63(3 \mathrm{H} . \mathrm{s}), 3.46(3 \mathrm{H}$. s). $0.92(9 \mathrm{H} . \mathrm{s}) .0 .07(6 \mathrm{H}, \mathrm{s}):{ }^{13} \mathrm{C} \operatorname{NMR}\left(75 \mathrm{MHz}, \mathrm{CDCl}_{3}\right) \hat{\delta}$ $150.8,142.7,138.9,123.6,117.5,113.5,98.8,95.2,63.9$. $57.9,56.2,25.9,18.3 .-5.3:$ IR (neat) $2952.1570,1479,1262$, $1158,1093,1011,954,842,675 \mathrm{~cm}^{-1}:$ HRMS $m / 2\left(\mathrm{M}^{+}\right)$calcd for $\mathrm{C}_{1}: \mathrm{H}_{2} \mathrm{O}_{5} \mathrm{BrSi}: 420.0968$. Found: 420.0970 .

[3-(3,7-Dimethoxymethoxyocta-2,6-dienyl)-4,5-dimethoxyphenyl]methanol (9). To a solution of $8(0.80 \mathrm{~g}, 1.9 \mathrm{mmol})$ in dry THF $(10 \mathrm{~mL})$ at $-78^{\circ} \mathrm{C}$ were added $n-B u L i(0.9 \mathrm{~mL} .2 .5 \mathrm{M}$ in hexane. $2.3 \mathrm{mmol}$ ), and the reaction mixture was stirred for $1 \mathrm{~h}$. To the reaction nuxture was added geranyl bronide (0.618 g. $2.85 \mathrm{mmol}$ ) slowly via șringe and continued the reaction at the same temperature for $2 \mathrm{~h}$. Then. the reaction mixture was stirred at $0^{\circ} \mathrm{C}$ for $12 \mathrm{~h}$ and was quenched by the addition of water $(30 \mathrm{~mL})$ and extracted with EtOAc $(3 \times 30$ $\mathrm{mL}$ ). The organic layer was washed with saturated $\mathrm{NH}_{4} \mathrm{Cl}$ solution $(30 \mathrm{~mL})$ and then dried over anhyydrous $\mathrm{Na}_{2} \mathrm{SO}_{4}$ and concentrated under reduced pressure to afford crude residue. To the solution of crude residue in THF ( $10 \mathrm{~mL})$ was added TBAF (1.9 $\mathrm{mL}, 1.9 \mathrm{mmol} .1 .0 \mathrm{M}$ in THF) at $0^{\circ} \mathrm{C}$ and stirred at room temperature for $3 \mathrm{~h}$. The reaction mixture was quenched by the addition of saturated $\mathrm{NH}_{4} \mathrm{Cl}$ solution $(30 \mathrm{~mL})$ and extracted with $\mathrm{EtOAc}(3 \times 30 \mathrm{~nL})$. The organic layer was washed with water $(30 \mathrm{~mL})$ and dried over anhy drous $\mathrm{Na}_{2} \mathrm{SO}_{4}$ and concentrated under reduced pressure to give the crude residue. The resulting cnude residue was purified by column chromatography with hexane/EtOAc (4:1) afforded $9(0.423 \mathrm{~g} .61 \%)$ as an oil. ${ }^{\mathrm{j}} \mathrm{H} \mathrm{NMR}\left(300 \mathrm{MHz} . \mathrm{CDCl}_{3}\right) \delta 6.98(\mathrm{HH}, \mathrm{s}) .6 .81$ (1H. s), 5.28 (lH. t. $J=7.2 \mathrm{~Hz}$ ). $5.16(2 \mathrm{H} . \mathrm{s}) .5 .07(3 \mathrm{H} . \mathrm{s}) .4 .55(2 \mathrm{H} . \mathrm{s}) .3 .57$ (3H. s), 3.47 (3H. s), 3.40 (2H. d, $J=7.2 \mathrm{~Hz}) 2.14-1.98(4 \mathrm{H}$, m). $1.69(3 \mathrm{H}, \mathrm{s}), 1.65(3 \mathrm{H}, \mathrm{s}) .1 .57(3 \mathrm{H}, \mathrm{s}):{ }^{13} \mathrm{C} \mathrm{NMR}(75 \mathrm{MHz}$. $\left.\mathrm{CDCl}_{2}\right) \delta 1+9.6,1+3.9,137.0,136.1,135.9,131.2,124.1,122.4$, $121.4,112.6,98.9 .94 .9 .64 .8,57.3,56.0 .39 .6 .28 .3 .26 .5$. $25.5,17.5,16.0$ : IR (neat) $3456,2924,1597,1447,1297,1155$. 
$1042 \mathrm{~cm}^{-1}$ : HRMS $m / z\left(\mathrm{M}^{-}\right)$calcd for $\mathrm{C}_{21} \mathrm{H}_{3 \leq} \mathrm{O}_{5}: 364.2250$. Found: $36+2252$.

3,4-Bis(methoxymethoxy)-5-(3,7-diemthylocta-2,6-dienyl) benzaldehyde (10). To a stirred solution of $9(0.150 \mathrm{~g}, 0.4$ mmol) in dry methylene chloride $(10 \mathrm{~mL})$ was added DessMartin periodinane (DMP) $(0.17+\mathrm{g} .0 .4 \mathrm{mmol})$ at $0{ }^{\circ} \mathrm{C}$. and the reaction mixture was stirred at room temperature for $3 \mathrm{~h}$. The reaction mixture was quenched with saturated sodium bicarbonate $(30 \mathrm{~mL}$ ) and extracted with methy lene chloride (3 $\times 30 \mathrm{~mL}$ ). The organic layers were washed with saturated sodium thiosulfate $(20 \mathrm{~mL})$, water $(30 \mathrm{~mL})$ and dried over anhy'drous $\mathrm{Na}_{2} \mathrm{SO}_{+}$and concentrated under reduced pressure to give the crude residue. The resulting crude residue was purified by column chromatography with hexane/EtOAc (4:1) to afford $10(0.145 \mathrm{~g} .98 \%)$ as an oil: ${ }^{1} \mathrm{H}$ NMR $(300 \mathrm{MHz}$. $\left.\mathrm{CDCl}_{3}\right) \delta 9.83$ (1H. s). 7.49 (1H. d. $\left.J=2.0 \mathrm{~Hz}\right), 7.36(1 \mathrm{H} . \mathrm{d}, J=$ $2.0 \mathrm{~Hz}) .5 .30(\mathrm{lH}, \mathrm{t} . J=7.0 \mathrm{~Hz}) .5 .22(2 \mathrm{H}, \mathrm{s}) .5 .20(2 \mathrm{H} . \mathrm{s})$. $5.10-5.01(1 \mathrm{H}, \mathrm{m}), 3.57(3 \mathrm{H}, \mathrm{s}), 3.47(3 \mathrm{H} . \mathrm{s}), 3.40(2 \mathrm{H} . \mathrm{d}, J=$ $7.0 \mathrm{~Hz}) \cdot 2.14-2.01(+\mathrm{H} . \mathrm{m}) .1 .70(3 \mathrm{H} . \mathrm{s}) .1 .64(3 \mathrm{H} . \mathrm{s}) .1 .57$ (3H. s); ${ }^{13} \mathrm{C}$ NMR $\left(75 \mathrm{MHz} . \mathrm{CDCl}_{3}\right) \delta 191.4,152.6 .149 .5$. $137.2,136.3,132.4 .131 .5$. 126.3. 124.1. 121.5. 108.4, 98.8. $57.6 .55 .8,39.6,28.1,26.5,25.6 .17 .6 .16 .1$ IR (neat) 3464. 2933. 1691, 1587. 1460, 1297. 1153.1078, 959.767.624, 476 $\mathrm{cm}^{-1}:$ FAB-HRMS $m / z[\mathrm{M}+\mathrm{H}]^{+}$calcd for $\mathrm{C}_{z 1} \mathrm{H}_{31} \mathrm{O}_{5}: 363.2171$. Found: 363.2167.

Chalcone 11. To a solution of $+(0.120 \mathrm{~g} .0 .+\mathrm{mmol})$ and 10 $(0.13 \mathrm{lg} .0 .4 \mathrm{mmol})$ in ethanol $(10 \mathrm{~mL})$ was added potassium hydroxide $(0.203 \mathrm{~g} .3 .6 \mathrm{mmol})$ at $0^{\circ} \mathrm{C}$. and the reaction was stirred at room temperature for $48 \mathrm{~h}$. Evaporation of ethanol. addition of $2 \mathrm{~N} \mathrm{HCl}$ solution $(50 \mathrm{~mL})$. and extraction with ethy' acetate $(3 \times 50 \mathrm{~mL})$, washing with brine $(50 \mathrm{~mL})$. drying over anhydrous $\mathrm{MgSO}_{+}$. and removal of the solvent followed by flash column chromatography on silica gel using hexane/ ethylacetate (20:1) afforded $11(0.139$ g. $57 \%)$ as an oil: ${ }^{1} \mathrm{H}$ $\operatorname{NMR}\left(300 \mathrm{MHz}, \mathrm{CDCl}_{3}\right) \delta$ o $13.42(1 \mathrm{H}, \mathrm{s}), 7.77(1 \mathrm{H}, \mathrm{d} . J=15.0$ Hz). 7.73 (IH. d. $J=8.7 \mathrm{~Hz}$ ). 7.43 (lH. d. $J=15.0 \mathrm{~Hz}$ ). 7.28 (1H. s). $7.12(1 \mathrm{H}, \mathrm{s}) .6 .67(1 \mathrm{H}, \mathrm{d}, J=8.7 \mathrm{~Hz}), 5.33 .5 .22(2 \mathrm{H}$, m). $5.25(2 \mathrm{H} . \mathrm{s}) .5 .22(2 \mathrm{H} . \mathrm{s}) .5 .15(2 \mathrm{H} . \mathrm{s}) .5 .15-5.01(2 \mathrm{H} . \mathrm{m})$. $3.59(3 \mathrm{H}, \mathrm{s}) .3 .52(3 \mathrm{H}, \mathrm{s}), 3.46(3 \mathrm{H}, \mathrm{s}) .3 .46-3.39(4 \mathrm{H} . \mathrm{m})$. $2.21-1.93(8 \mathrm{H} . \mathrm{m}), 1.79(3 \mathrm{H}, \mathrm{s}), 1.72(3 \mathrm{H}, \mathrm{s}), 1.65(3 \mathrm{H}, \mathrm{s}) .1 .62$ (3H.s). 1.58 (3H. s). 1.55 (3H. s): ${ }^{13} \mathrm{C} \mathrm{NMR}\left(75 \mathrm{MHz}, \mathrm{CDCl}_{3}\right)$ ò 192.2. 163.2. 160.6, 149.9, 147.1, 144.2, 136.9. 136.4. $135.1,131.4,131.0,130.7,128.7,124.3,124.2,124.0,121.8$. $119.4,118.4,114.9 .113 .5,104.7 .98 .9 .95 .1 .93 .7 .57 .4,56.2$, $56.0 .39 .7,28.2,26.6 .25 .6,21.7 .17 .6 .17 .5,16.1 .16 .0$; IR (neat) $2961,2918,2852.207+, 1728,1633,1582,1+87,1+33$. 1371. 1264, 1155, 1109. 1078. 1046,960.795,661,609 $\mathrm{cm}^{-1}$ : FAB-HRMS $m / z[\mathrm{M}+\mathrm{H}]^{-}$calcd for $\mathrm{C}_{41} \mathrm{H}_{5} \mathrm{O}_{8}: 677.4053$. Found: 677.4056 .

Porepensin (1). To a solution of chalcone $11(0.050 \mathrm{~g} .0 .1$ mmol) in methanol $(5 \mathrm{~mL})$ was added $\mathrm{c}-\mathrm{HCl}(5 \mathrm{drops})$ at $0^{\circ} \mathrm{C}$. and the reaction mixture was stirred at room temperature for $12 \mathrm{~h}$. The reaction mixture was diluted with saturated $\mathrm{NaHCO}_{3}$ solution $(30 \mathrm{~mL})$ and extracted with EtOAc $(3 \times 30 \mathrm{~mL})$. Removal of solvent at reduced pressure left an oily residue. which was then purified by column chromatography on silica gel with hexane/EtOAc (2:1) to give $1(0.02+\mathrm{g} .60 \%)$ as an oil: ${ }^{1} \mathrm{H}$ NMR (300 MHz. $\mathrm{CDCl}_{3}$ ) ô 13.89 (lH. s). 7.72 (lH. d.
$J=15.3 \mathrm{~Hz}) .7 .66(1 \mathrm{H}, \mathrm{d} . J=9.0 \mathrm{~Hz}) .7 .36(1 \mathrm{H} . \mathrm{d} . J=15.3$ Hz). $7.06(1 \mathrm{H}, \mathrm{s}) .6 .94(\mathrm{lH}, \mathrm{s}) .6 .39(1 \mathrm{H}, \mathrm{d} . J=9.0 \mathrm{~Hz})$. $5.34-5.26(2 \mathrm{H}, \mathrm{m}), 5.14-5.01(2 \mathrm{H}, \mathrm{m}) .3 .46(2 \mathrm{H} . \mathrm{d} . J=7.2$ Hz). $3.37(2 \mathrm{H}, \mathrm{d}, J=7.2 \mathrm{~Hz}) .2 .20-2.01(8 \mathrm{H} . \mathrm{m}), 1.80(3 \mathrm{H} . \mathrm{s})$, $1.75(3 \mathrm{H}, \mathrm{s}) .1 .66(3 \mathrm{H}, \mathrm{s}), 1.65(3 \mathrm{H}, \mathrm{s}), 1.59(3 \mathrm{H}, \mathrm{s}), 1.58(3 \mathrm{H}$. s); ${ }^{12} \mathrm{C} \mathrm{NMR}\left(75 \mathrm{MHz} . \mathrm{CDCl}_{5}\right) \delta 192.3 .163 .8,161.8 .145 .1$, $144.8,144.2,139.4,139.0,132.1,132.0,129.3,127.8,127.3$, 123.7, 123.6, 121.1, 120.8, 117.9. 114.2. 113.9, 112.4, 107.9. $39.7,39.6 .29 .3 .26 .4$. $26.325 .7,25.6 .21 .7 .17 .7$. 17.6, 16.2 , 16.1: $\mathbb{R}$ (neat) 3408, 2918, 2357, 1609. 1492, 1440, 1378 . $1277,1105.1040,976,846,801,739,622 \mathrm{~cm}^{-1}$ : FAB-HRMS $m / 2[\mathrm{M}+\mathrm{H}]^{+}$calcd for $\mathrm{C}_{35} \mathrm{H}_{45} \mathrm{O}_{5}: 545.3267$. Found: 545.3264 .

Acknowledgments. This work was supported by the Korea Research Foundation Grant funded by the Korean Government (KRF-2008-313-C00476).

\section{References}

1. (a) Kumazawa, S:; Ueda, R.: Hamasaka, T:: Fukumoto, S.; Fuijimoto, T.; Nakayama, T. J. Agric. Food Chem. 2007, 55, 7722. (b) Miranda, C. L.; Stevens, .T. F.; Ivanov, V; McCall, M:; Frei, B.; Deinzer, M. L.; Buhler, D. R. J. Agric. Food Chem. 2000, 48, 3876. (c) Vogel, S.; Heilmann, J. J. Kat. Prod. 2008. 71, 1237. (d) Rodrigulez, R. I.; Miranda, C. L.; Stevens, I. F.; Deinzer, M. L.; Buhler, D. R. Food Chen. Toxicol 2001, 39 , 437. (e) Chi, Y. S.; Jong. H. G.; Son, K. H.; Chang, H. W.; Kang, S. S.; Kim, H. P. Biachem. Phamtacol. 2001, 62, 1185. (f) Miranda, C. L.: Aponso, G. L. M.: Stevens, I. F.: Deinzer, M. L.; Buhler, D. R. Cancer Lett. 2000, 1+9, 21.

2. Inamori, Y.; Baba, K.; Tsujibo, H.; Taniguchi, M.; Nakata, K.; Kozawa, M. Chem. Pham. Bull. 1991, 39, 1604.

3. Tayasinghe, L.: Balasooriya, B. A. I. S.; Padmini, W. C.: Hara, N.; Fulimoto, Y. Phytochemism 2004, 65, 1287.

4. Kimura, Y; Baba, K. Int. J. Cancer 2003, 106, 429.

5. Kimura, Y. Taniguchi, M.; Baba, K. Planta led 2004, 70, 211 .

6. Akihisa, T.: Tokulda, H.; Hasegawa, D.: Ukiya, M.: Kimura, Y.; Enjo, F.: Suzuki, T.: Nishino, H. J. Kat. Prod. 2006, 69, 38.

7. Rosselli, S.; Bruno, M.; Maggio, A.; Bellone, G.; Formisano, C.; Mattia, C. A.; Di Micco, S. Bifulco, G. Eum. J. Org. Chent. 2007, 2504.

8. Murakami, S.; Kịima, H.: Isobe, Y.; Muramatsu, M.; Aihara, H.; Otomo, S.; Baba, K.: Kozawa, M. I. Pham. Phamacol. 1990. 42,723

9. Enoki, T.; Ohnogi, H.; Nagamine, K.; Kudo, Y.; Sugivama, K.; Tanabe, M.: Kobayashi, E.: Sagawa, H.: Kato, I. J. Agric. Food Chem. 2007, 55, 6013

10. Abegaz, B. M.; Ngadjui, B. T:; Dongo, E.; Bezabih, M.-T. Curr Org. Chem. 2000, $4,1079$.

11. Nikaido, T:: Ohmoto, T:; Kinoshita, T:: Sankawa, U.: Delle Monache, F.: Botta, B.: Tomimori, T:; Miyaichi, Y.: Shirataki, Y: Yokoe, I, Komatsu, M. Chem. Pham. Bull. 1989,37, 1392.

12. (a) Abegaz, B. M.; Ngadjui, B. T; Dongo, E.; Ngameni, B:; Nindi, M. N.; Bezabih, M. Phytochentistn 2002, 59, 877. (b) Vouffo, B.: Hussain, H.; Eyong, K. O.; Dongo, E.; Folefoc, G. N.; Nkengfack, A. E.: Krohn, K. Biochem. System. Ecol. 2008 , 36,730

13. (a) Lee, Y. R.; Li, X.; Kim, J. H. J. Org. Chem. 2008, 73, 4313. (b) Lee, Y. R.; Xia, L. Swithesis 2007, 3240 . (c) Lee, Y. R.: Kim, D. H. Symhesis 2006, 603 .

14. Huang, C.; Zhang, Z.; Li, Y. J. Nat. Prod. 1998, 61, 1283.

15. Boukouvalas, J.; Loach, R. P. J. Ong. Chem. 2008, 73, 8109

16. Tohma, H.: Kita, Y. Adn. Sinth. Catal 2004, 3t6, 111. 